\title{
Calcul de l'agitation de houle sur un cluster de PCs à l'aide de la méthode de décomposition de domaines
}

\author{
Bainian ZHANG ${ }^{1}$, Bruno BADER ${ }^{1}$, Philippe SERGENT ${ }^{1}$, \\ Pierre DEBAILLON ${ }^{1}$ \\ ${ }^{1}$ Centre d'Etudes Techniques Maritimes Et FLuviale, Laboratoire d'Hydraulique \\ Numérique, 2 boulevard Gambetta, 60200 Compiègne, France. \\ Bainian.zhang@developpement-durable.gouv.fr
}

\section{Résumé :}

Le calcul d'agitation de houle par des modèles aux éléments finis est classiquement limité par la taille du système à résoudre. La taille du système est d'autant plus grande que le domaine physique est grand et que la période de la houle est petite. Les machines actuelles qui sont limitées par leur taille mémoire ne peuvent calculer l'agitation de houle pour des domaines de surface supérieure à quelques kilomètres carrés. C'est la raison pour laquelle nous avons parallélisé le code d'agitation de houle REFONDE® sur un cluster de PCs à l'aide de la méthode de décomposition de domaines et la méthode FETI-DP. Nous montrons les gains obtenus : nombre de nœuds multiplié par 4, temps de calcul divisé par 2. Les gains maximaux sont relevés pour six machines parallèles.

\begin{abstract}
:
Computation of water waves in harbour areas with finite element methods is usually limited by the size of system to solve. The size of this system is all the larger than harbour area is large and wave period is small. Present computers that are limited by their memory size can not calculate water waves when harbour areas exceed several square kilometres. That is why we parallelized water waves model REFONDE® on a PC cluster with the domain decomposition method and FETI-DP method. We show the following gains: nodes number multiplied by 4 , computation time divided by 2 . Maximum time reduction is found with six parallel machines.
\end{abstract}

Mots-clés :

Agitation - Houle - Elements finis - REFONDE - Décomposition de domaines FETI-DP - Cluster - Parallélisation - Découpage 


\section{Introduction}

Le calcul de l'agitation de la houle revêt une forte importance en particulier pour l'aménagement portuaire. De nombreux travaux ont eu lieu ces dernières années pour améliorer la prise en compte des phénomènes physiques ainsi que le traitement des conditions aux limites (SERGENT et al., 2002). Il s'avère cependant que même aujourd'hui les capacités de calcul ne permettent pas de traiter l'agitation avec les modèles standard dans les grands bassins portuaires français. C'est la raison pour laquelle les travaux prioritaires portent désormais en ce qui concerne l'agitation de houle sur les capacités de calcul et la parallélisation des codes. Les premiers résultats (LEFRANCOIS et al., 2004) ont prouvé la possibilité d'appliquer la méthode de décomposition de domaines au code d'agitation de houle REFONDE®. Ces travaux préliminaires ont été menés sur un solveur basé sur un stockage partiel des matrices du type SKYLINE. Les applications portaient sur des cas à 20 mille degrés de liberté. Dans ce papier, une nouvelle méthode de décomposition de domaines est testée, la méthode FETI-DP. Des applications avec jusqu'à 28 millions de degrés de liberté sont présentées.

\section{Modèle d'agitation de houle}

Le code de calcul REFONDE® est basé sur la résolution de l'équation de pente douce (BERKHOFF, 1972) par la méthode des éléments finis.

$$
\nabla\left(c c_{g} \nabla \phi\right)+k^{2} c c_{g} \phi=0
$$

où $c, c_{g}, k$ et $\phi$ sont respectivement la vitesse de phase, la vitesse de groupe, le nombre d'onde et le potentiel complexe des vitesses.

Après introduction des conditions aux limites, une discrétisation avec un élément fini linéaire de type triangulaire (T3) conduit au système suivant :

$[K]\{\phi\}=\{F\}$

avec $[K]$ la matrice rigidité, $\{\phi\}$ le vecteur des inconnues nodales du potentiel des vitesses et $\{F\}$ le vecteur des sollicitations.

\section{Méthode de décomposition de domaine}

\subsection{Formulation}

Nous utilisons la méthode FETI-DP « dual - primal unified finite element tearing and interconnecting » (FARHAT et al., 2001). Notons $\Omega$ le domaine de calcul et $\Omega^{\mathrm{s}}$ sa décomposition en $N_{s}$ sous-domaines avec les interfaces correspondantes. On note $K^{\mathrm{s}}, \phi^{\mathrm{s}}$ et $f^{\mathrm{s}}$ la matrice de rigidité, le vecteur des potentiels de vitesse et le vecteur des sollicitations associés avec le sous-domaine $\Omega^{\mathrm{s}}$ respectivement. Ces quantités sont réparties comme suit : 
$K^{s}=\left[\begin{array}{ll}K_{i i}^{s} & K_{i f}^{s} \\ K_{i f}^{s *} & K_{f f}^{s}\end{array}\right], \phi^{s}=\left[\begin{array}{l}\phi_{i}^{s} \\ \phi_{f}^{s}\end{array}\right], f^{s}=\left[\begin{array}{l}f_{i}^{s} \\ f_{f}^{s}\end{array}\right]$

où les indices $i$ et $f$ désignent le domaine interne et les degrés de liberté à la frontière respectivement. * désigne l'opération hermitienne. On partitionne encore une fois la composante $\phi_{f}^{s}$ comme suit :

$\phi_{f}^{s}=\left[\begin{array}{c}\phi_{f_{r}}^{s} \\ \phi_{f_{c}}^{s}\end{array}\right]$

où l'indice $\mathrm{c}$ désigne le degré de liberté attaché aux « coins » de la décomposition de maillage et l'indice $\mathrm{r}$ désigne le "reste » des degrés de liberté de l'interface. Les coins issus de la décomposition de domaine sont définis de deux façons :

- Des points appartenant à plus de deux sous-domaines (D1)

- Des points appartenant au début et à la fin d'un côté d'un sous-domaine (D2)
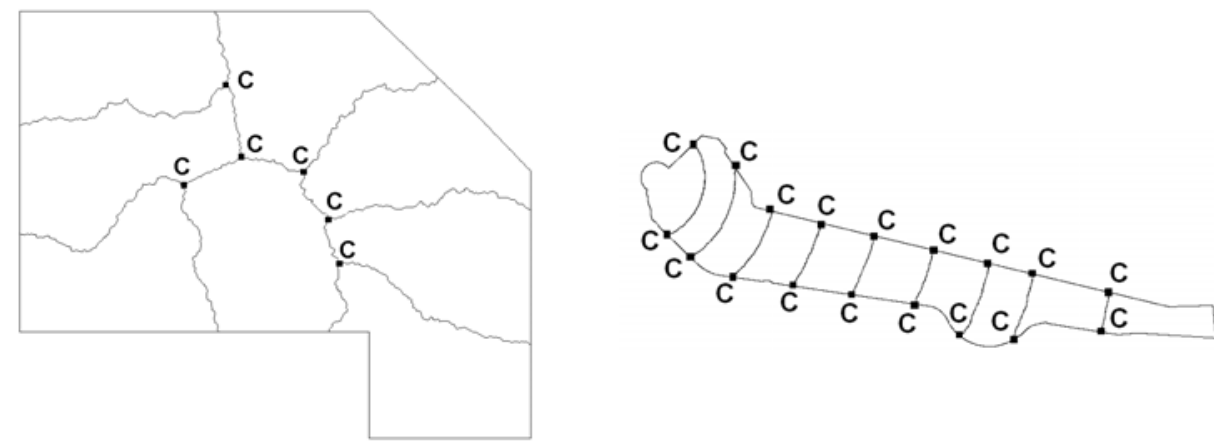

Figure 1. Définition D1 d'un point de coin et définition D2 d'un point de coin.

La continuité du potentiel des vitesses aux interfaces des sous-domaines est écrite sous la forme:

$\phi_{f}^{s}-\phi_{f}^{q}=0 \quad \operatorname{sur} \Omega^{\mathrm{s}} \cap \Omega^{\mathrm{q}}$ ou $\sum_{s=1}^{s=N_{s}} B^{s} \phi^{s}=0$

où $B^{s}$ est une matrice booléenne signée. La méthode FETI consiste à introduire des multiplicateurs de Lagrange $\lambda$ pour assurer la condition de continuité (5) (TOSELLI \& WIDLUND, 2005, page 145) et à employer l'algorithme du type CGS pour déterminer les valeurs de ces multiplicateurs. Les inconnues aux points coins sont définies au niveau global tandis que les autres inconnues sont définies au niveau du sous-domaine. Ainsi $K^{\mathrm{s}}, \phi^{s}$ et $f^{\mathrm{s}}$ sont repartitionnés de la façon suivante :

$$
K^{s}=\left[\begin{array}{ll}
K_{r r}^{s} & K_{r c}^{s} \\
K_{r c}^{s *} & K_{c c}^{s}
\end{array}\right], \phi^{s}=\left[\begin{array}{l}
\phi_{r}^{s} \\
\phi_{f_{c}}^{s}
\end{array}\right], f^{s}=\left[\begin{array}{c}
f_{r}^{s} \\
f_{f_{c}}^{s}
\end{array}\right]
$$


avec $\phi_{r}^{s}=\left[\begin{array}{c}\phi_{i}^{s} \\ \phi_{f_{r}}^{s}\end{array}\right], f_{r}^{s}=\left[\begin{array}{c}f_{i}^{s} \\ f_{f_{r}}^{s}\end{array}\right]$ et $\phi_{f_{c}}^{s}$ formé de $N_{c}$ composantes correspondant aux nœuds de coins. Pour chaque sous-domaine $\Omega^{\mathrm{s}}$ sont définies les matrices booléennes signées $B_{r}^{s}$ et $B_{c}^{s}$ avec le signe de l'égalité déterminée par convention telles que :

$B_{r}^{s} \phi_{r}^{s}= \pm \phi_{f_{r}}^{s}$ et $B_{c}^{s} \phi_{c}^{s}=\phi_{f_{c}}^{s}$

En utilisant les notations (6)-(7), les équations d'équilibre du sous-domaine s'écrivent avec la condition de continuité d'interface :

$$
\left\{\begin{array}{c}
K_{r r}^{s} \phi_{r}^{s}+K_{r c}^{s} B_{c}^{s} \phi_{c}=f_{r}^{s}-B_{r}^{s T} \lambda \\
\sum_{s=1}^{s=N_{s}} B_{c}^{s T} K_{r c}^{s T} \phi_{r}^{s}+\sum_{s=1}^{s=N_{s}} B_{c}^{s T} K_{c c}^{s} B_{c}^{s} \phi_{c}=\sum_{s=1}^{s=N_{s}} B_{c}^{s T} f_{f_{c}}^{s}=f_{c} \\
\sum_{s=1}^{s=N_{s}} B_{r}^{s} \phi_{r}^{s}=0
\end{array}\right.
$$

\subsection{Résolution}

La première équation de (8) donne le déplacement pour tous les points excepté les points coins :

$$
\phi_{r}^{s}=K_{r r}^{s-1}\left(f_{r}^{s}-B_{r}^{s T} \lambda-K_{r c}^{s} B_{c}^{s} \phi_{c}\right)
$$

Les inconnues sont les multiplicateurs de Lagrange $\lambda$ rattachés aux points frontières ainsi que les déplacements $\phi_{c}$.

Substituant l'équation (9) dans les deux dernières équations du système (8) on obtient le dual - primal problème :

$\left[\begin{array}{ll}F_{I_{r r}} & F_{I_{r c}} \\ F_{I_{r c}}^{*} & \bar{K}_{c c}\end{array}\right]\left[\begin{array}{l}\lambda \\ \phi_{c}\end{array}\right]=\left[\begin{array}{l}d_{r} \\ \bar{f}_{c}\end{array}\right]$

où $\quad F_{I_{r r}}=\sum_{s=1}^{s=N_{s}} B_{r}^{s} K_{r r}^{s-1} B_{r}^{s T}, \quad F_{I_{r c}}=\sum_{s=1}^{s=N_{s}} B_{r}^{s} K_{r r}^{s-1} K_{r c}^{s} B_{c}^{s}, \quad K_{c c}=\sum_{s=1}^{s=N_{s}} B_{c}^{s T} K_{c c}^{s} B_{c}^{s}$,

$\bar{K}_{c c}=K_{c c}-\sum_{s=1}^{s=N_{s}}\left(K_{r c}^{s} B_{c}^{s}\right)^{T} K_{r r}^{s-1}\left(K_{r c}^{s} B_{c}^{s}\right), \quad d_{r}=\sum_{s=1}^{s=N_{s}} B_{r}^{s} K_{r r}^{s-1} f_{r}^{s}$,

$\bar{f}_{c}=f_{c}-\sum_{s=1}^{s=N_{s}} B_{c}^{s T} K_{r c}^{s T} K_{r r}^{s-1} f_{r}^{s}$

Le problème est dit dual - primal car il se réfère aux multiplicateurs de Lagrange $\lambda$ (problème dual) et aux degrés de libertés du problème primal : les potentiels de vitesse $\phi_{c}$. 


\section{$\underline{3.3 \text { Méthodes d'inversion }}$}

Les matrices $K_{r r}^{s}$ sont décomposées par une méthode directe sur chaque sousdomaine avec une résolution LU et stockage des matrices du type MORSE.

Le problème dual est résolu par une méthode itérative du type CGS appelée BICGSTAB.

\section{Cas-test : Port 2000 au Havre}

\subsection{Le cluster}

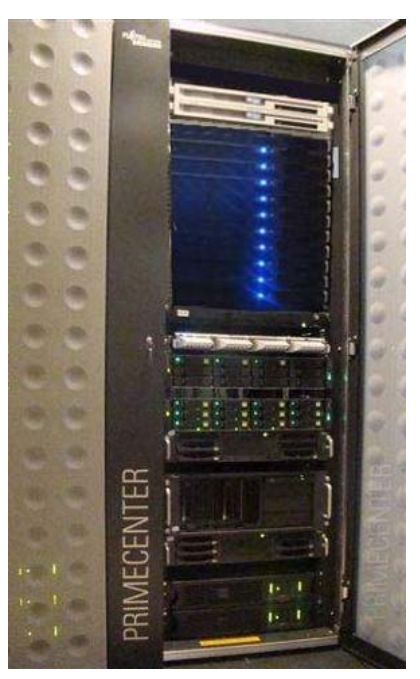

Figure 2. Photo du Cluster du CETMEF.

Le cluster du CETMEF est un cluster Beowulf de classe II, constitué de 24 nœuds bi processeurs bi cœurs utilisant un réseau Myrinet pour les échanges de calcul et un réseau Gigabyte ethernet pour les messages de service. Il dispose d'une mémoire totale de 100Go et est relié à une baie de disques de 1 To en raid 5 pour le stockage. Le cluster offre une puissance totale de 160 Gflops (160 milliards d'opérations en virgule flottante par seconde).

La gestion des travaux sur le cluster est réalisé par l'ordonnanceur Sun Grid Engine et les programmes parallèles, essentiellement en Fortran, tirent parti de la bibliothèque MPI (Message Passing Interface) et du compilateur PGI.

\subsection{Programme de découpage}

Le découpage du domaine est réalisé à l'aide de la bibliothèque Metis (KAPYRIS et KUMAR, 1998). Metis est un algorithme de partitionnement de graphe développé à l'université du Minnesota. Sur le principe, le maillage initial de Delaunay est converti en un graphe, qui est ensuite transformé séquentiellement en graphe plus petit donnant toujours une assez bonne représentation du graphe initial. Le plus petit graphe est ensuite partitionné et donne une partition qui expansée donne la partition finale. Le partitionnement est réalisé en essayant d'équilibrer le nombre d'éléments par parties. 


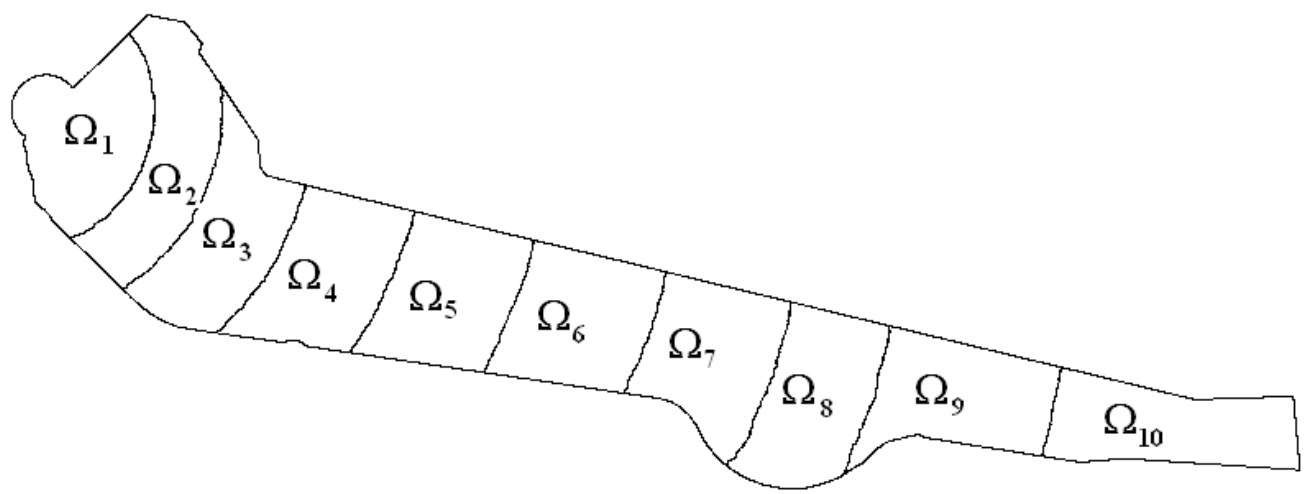

Figure 3. Découpage du Port 2000 au Havre en dix sous-domaines.

D'autres programmes de découpage peuvent être utilisés (LÖHNER R., 2001, page 330).

\section{$\underline{4.3 \text { Analyse des performances }}$}

Un régime de houle régulière de période de $10 \mathrm{~s}$, de hauteur $0,5 \mathrm{~m}$ et de direction $280^{\circ}$ est choisi avec une hauteur de mer de 7,90 m.

Nous prenons les notations suivantes : Nnt est le nombre de nœuds, Nelt est le nombre d'éléments, $\mathrm{Np}$ est le nombre de machines égale au nombre Ns de sousdomaines.

Les tableaux 1 à 8 montrent le temps de résolution et le temps total pour des maillages de 200 mille nœuds à 14 millions de noeuds. La différence entre les deux temps est due aux temps de construction et d'assemblage des matrices. Ce temps d'assemblage diminue relativement au temps total lorsque la taille $\mathrm{du}$ problème augmente. Il passe très vite en deçà de $10 \%$ du temps total.

Sur notre cluster, le calcul passe en mémoire vive sur une machine jusqu'à environ 2 millions de nœuds. Au-delà le calcul utilise la mémoire SWAP et ralentit rapidement. Il faut utiliser schématiquement 5 machines pour un calcul à 10 millions de nœuds ou 10 machines pour un calcul à 20 millions de nœuds.

Pour le calcul avec la période de $10 \mathrm{~s}-7 \mathrm{~s}-4 \mathrm{~s}$, avec une densité de maillage de 10 nœuds par longueur d'onde, le nombre de nœuds nécessaire s'élève à 50 mille, 150 mille et 1 million respectivement. 


\begin{tabular}{crrrr}
\hline $\begin{array}{l}\text { Nombre } \\
\text { de PCs }\end{array}$ & $\begin{array}{l}\text { Nombre } \\
\text { de Points } \\
\text { Dual }\end{array}$ & $\begin{array}{l}\text { Nombre } \\
\text { itérations }\end{array}$ & $\begin{array}{l}\text { Temps de } \\
\text { résolution } \\
\text { (s) }\end{array}$ & $\begin{array}{l}\text { Temps } \\
\text { Total } \\
\text { (s) }\end{array}$ \\
\hline 1 & 0 & 1 & 16 & 26 \\
2 & 171 & 26 & 18,6 & 28 \\
4 & 623 & 67 & 17,6 & 26,8 \\
6 & 1034 & 118 & 18,4 & 27,5 \\
8 & 1387 & 213 & 22,6 & 31,6 \\
10 & 1793 & 306 & 28,2 & 37,5
\end{tabular}

\begin{tabular}{crrrr}
\hline $\begin{array}{l}\text { Nombre } \\
\text { de PCs }\end{array}$ & $\begin{array}{l}\text { Nombre } \\
\text { de Points } \\
\text { Dual }\end{array}$ & $\begin{array}{l}\text { Nombre } \\
\text { itérations }\end{array}$ & $\begin{array}{l}\text { Temps de } \\
\text { résolution } \\
\text { (s) }\end{array}$ & $\begin{array}{l}\text { Temps } \\
\text { Total } \\
\text { (s) }\end{array}$ \\
\hline 1 & 0 & 1 & 58 & 77 \\
2 & 235 & 27 & 54 & 74 \\
4 & 872 & 81 & 49 & 69 \\
6 & 1438 & 118 & 45 & 65 \\
8 & 1986 & 218 & 54 & 74 \\
10 & 2565 & 410 & 78 & 98
\end{tabular}

Tableau 1. Nnt=234 055, Nelt=464 704

\begin{tabular}{crrrr}
\hline $\begin{array}{l}\text { Nombre } \\
\text { de PCs }\end{array}$ & $\begin{array}{l}\text { Nombre } \\
\text { de Points } \\
\text { Dual }\end{array}$ & $\begin{array}{l}\text { Nombre } \\
\text { itérations }\end{array}$ & $\begin{array}{l}\text { Temps de } \\
\text { résolution } \\
(\mathbf{s})\end{array}$ & $\begin{array}{l}\text { Temps } \\
\text { Total } \\
\text { (s) }\end{array}$ \\
\hline 1 & 0 & 1 & 185 & 225 \\
2 & 345 & 32 & 153 & 192 \\
4 & 1284 & 82 & 120 & 160 \\
6 & 2037 & 161 & 128 & 167 \\
8 & 2843 & 223 & 130 & 170 \\
10 & 3598 & 443 & 187 & 227
\end{tabular}

Tableau 3. Nnt=932 809, Nelt $=1858813$

\begin{tabular}{crrrr}
\hline $\begin{array}{l}\text { Nombre } \\
\text { de PCs }\end{array}$ & $\begin{array}{l}\text { Nombre } \\
\text { de Points } \\
\text { Dual }\end{array}$ & $\begin{array}{l}\text { Nombre } \\
\text { itérations }\end{array}$ & $\begin{array}{l}\text { Temps de } \\
\text { résolution } \\
\text { (s) }\end{array}$ & $\begin{array}{l}\text { Temps } \\
\text { Total } \\
(\mathbf{s})\end{array}$ \\
\hline 1 & 0 & 1 & 1812 & 1990 \\
2 & 722 & 38 & 1427 & 1598 \\
4 & 2586 & 105 & 1126 & 1314 \\
6 & 4237 & 188 & 1014 & 1182 \\
8 & 5873 & 305 & 1042 & 1213 \\
10 & 7596 & 611 & 1429 & 1862
\end{tabular}

Tableau 2. Nnt=467 550, Nelt=930 231

\begin{tabular}{crrrr}
\hline $\begin{array}{l}\text { Nombre } \\
\text { de PCS }\end{array}$ & $\begin{array}{l}\text { Nombre } \\
\text { de Points } \\
\text { Dual }\end{array}$ & $\begin{array}{l}\text { Nombre } \\
\text { itérations }\end{array}$ & $\begin{array}{l}\text { Temps de } \\
\text { résolution } \\
\text { (s) }\end{array}$ & $\begin{array}{l}\text { Temps } \\
\text { Total } \\
\text { (s) }\end{array}$ \\
\hline 1 & 0 & 1 & 520 & 604 \\
2 & 469 & 33 & 469 & 550 \\
4 & 1797 & 107 & 363 & 456 \\
6 & 2933 & 140 & 290 & 370 \\
8 & 4015 & 261 & 350 & 433 \\
10 & 5147 & 466 & 477 & 558
\end{tabular}

Tableau 4. Nnt=1 864597, Nelt=3 719492

\begin{tabular}{cccccc}
\hline $\begin{array}{l}\text { Nombre } \\
\text { de PCs }\end{array}$ & $\begin{array}{l}\text { Nombre } \\
\text { de Points } \\
\text { Dual }\end{array}$ & $\begin{array}{l}\text { Nombre } \\
\text { itérations }\end{array}$ & $\begin{array}{l}\text { Temps de } \\
\text { résolution } \\
\text { (s) }\end{array}$ & $\begin{array}{l}\text { Temps } \\
\text { Total } \\
\text { (s) }\end{array}$ \\
\hline 1 & \multicolumn{1}{c}{-} & - & - & - \\
2 & 973 & 47 & 3334 & 3621 \\
4 & 3466 & 117 & 2311 & 2754 \\
6 & 5625 & 223 & 2285 & 2578 \\
8 & 7744 & 403 & 2526 & 2826 \\
10 & 9984 & 598 & 2844 & 3138
\end{tabular}

Tableau 5. Nnt=3 992 199, Nelt=7 970514 Tableau 6. Nnt=6 983488 , Nelt=13948 160

\begin{tabular}{ccccc}
\hline $\begin{array}{l}\text { Nombre } \\
\text { de PCs }\end{array}$ & $\begin{array}{l}\text { Nombre } \\
\text { de Points } \\
\text { Dual }\end{array}$ & $\begin{array}{l}\text { Nombre } \\
\text { itérations }\end{array}$ & $\begin{array}{l}\text { Temps de } \\
\text { résolution } \\
(\mathbf{s})\end{array}$ & $\begin{array}{l}\text { Temps } \\
\text { Total } \\
(\mathbf{s})\end{array}$ \\
\hline 1 & - & - & - & - \\
2 & - & - & - & - \\
4 & - & - & - & - \\
6 & 6538 & 213 & 2945 & 3387 \\
8 & 8971 & 346 & 3324 & 3798 \\
10 & 11570 & 714 & 4632 & 5012
\end{tabular}

\begin{tabular}{ccccc}
\hline $\begin{array}{l}\text { Nombre } \\
\text { de PCs }\end{array}$ & $\begin{array}{l}\text { Nombre } \\
\text { de Points } \\
\text { Dual }\end{array}$ & $\begin{array}{l}\text { Nombre'it } \\
\text { érations }\end{array}$ & $\begin{array}{l}\text { Temps de } \\
\text { résolution } \\
\text { (s) }\end{array}$ & $\begin{array}{l}\text { Temps } \\
\text { Total } \\
\text { (s) }\end{array}$ \\
\hline 1 & - & - & - & - \\
2 & - & - & - & - \\
4 & - & - & - & - \\
6 & - & - & - & - \\
8 & 11016 & 503 & 6392 & 7180 \\
10 & 14088 & 977 & 9782 & 10605
\end{tabular}

Tableau 7. Nnt=9 306 488, Nelt=18 590673 Tableau 8. Nnt=13962 756, Nelt=27925 510 


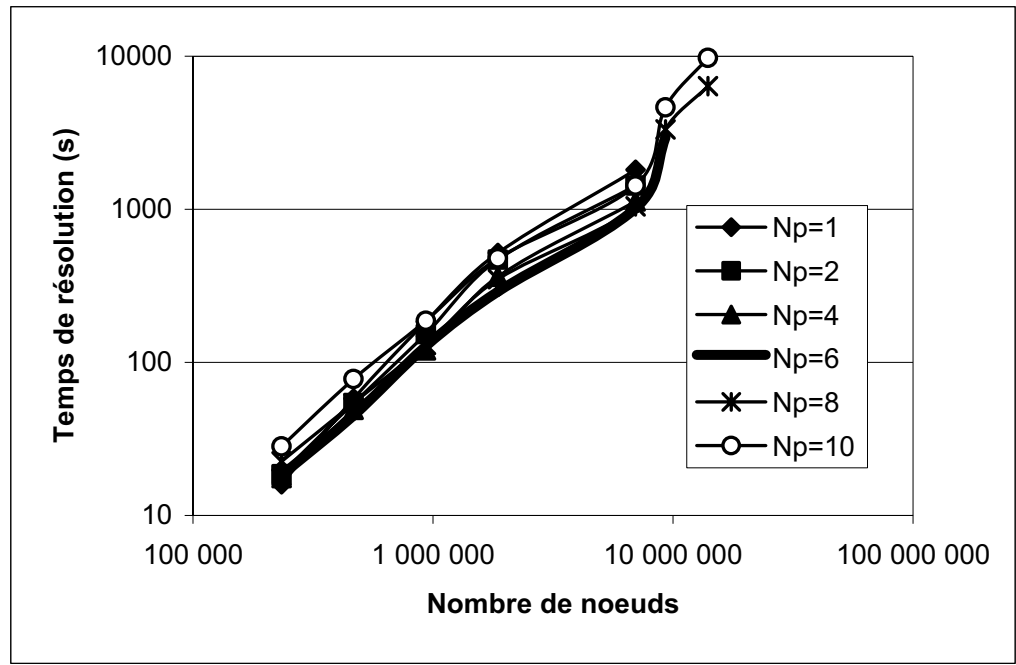

Figure 4. Evolution du temps de résolution en fonction du nombre de nouds.

La figure 4 montre que le temps de calcul reste en puissance $3 / 2$ du nombre de nœuds même en mode parallélisé. L'optimum en ce qui concerne le nombre de machines est trouvé à 6 machines.

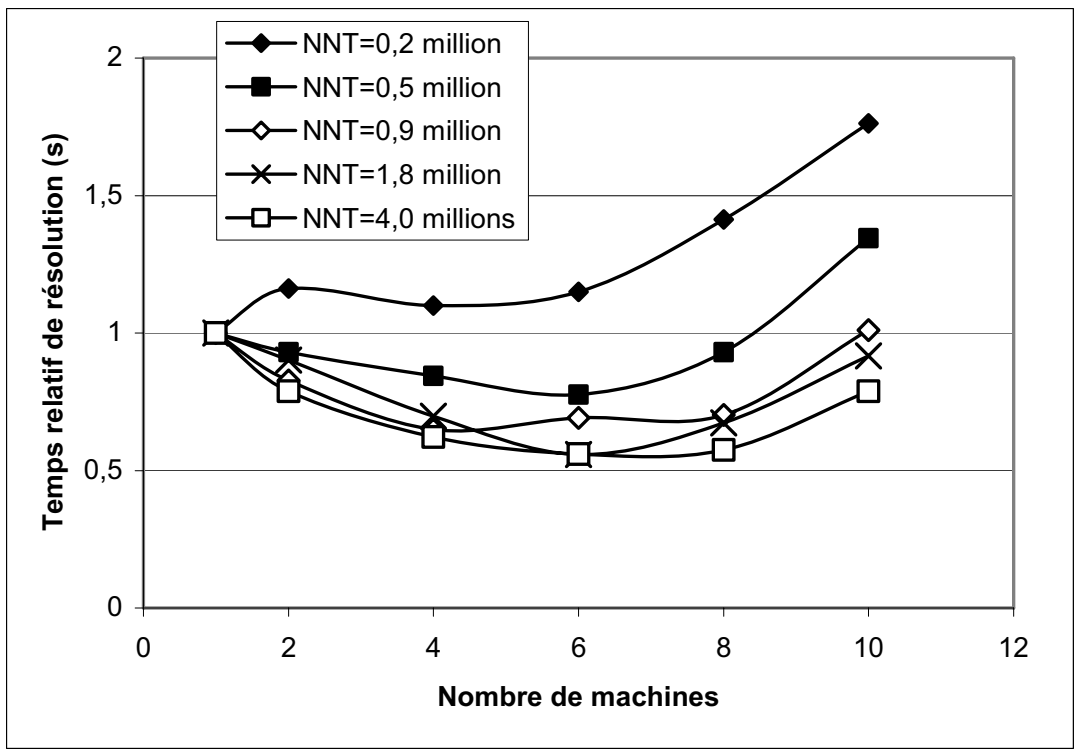

Figure 5. Temps de résolution relatif par rapport à un calcul mono-processeur.

La figure 5 montre un gain maximal d'un facteur 2 sur le temps de résolution. L'optimum est à 6 machines. Ce gain augmente avec la taille du problème. 


\subsection{Analyse des résultats}

Les résultats sont produits pour un régime de houle régulière de période de $3 \mathrm{~s}$, de hauteur $0,5 \mathrm{~m}$ et de direction $300^{\circ}$ avec une hauteur de mer de $7,90 \mathrm{~m}$. Une période courte est choisie afin de montrer un cas où un maillage dense est utile.

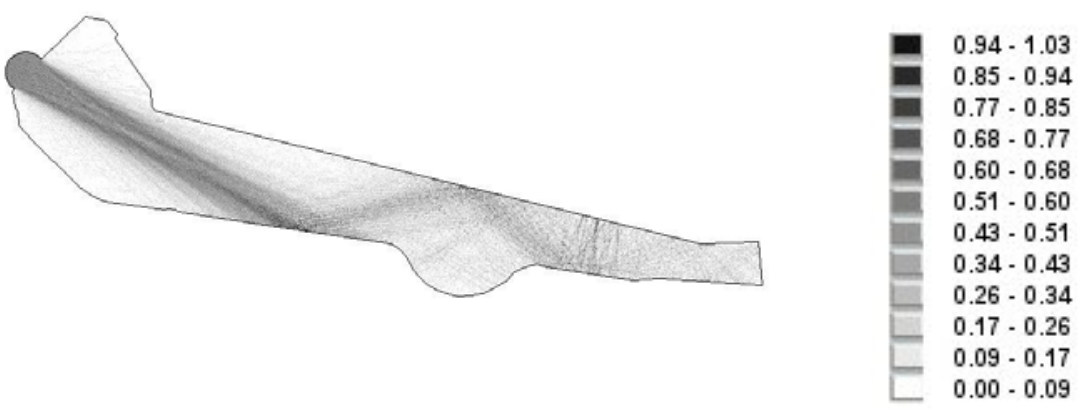

Figure 6. Hauteur de houle calculée sur un maillage de 500 mille næuds.

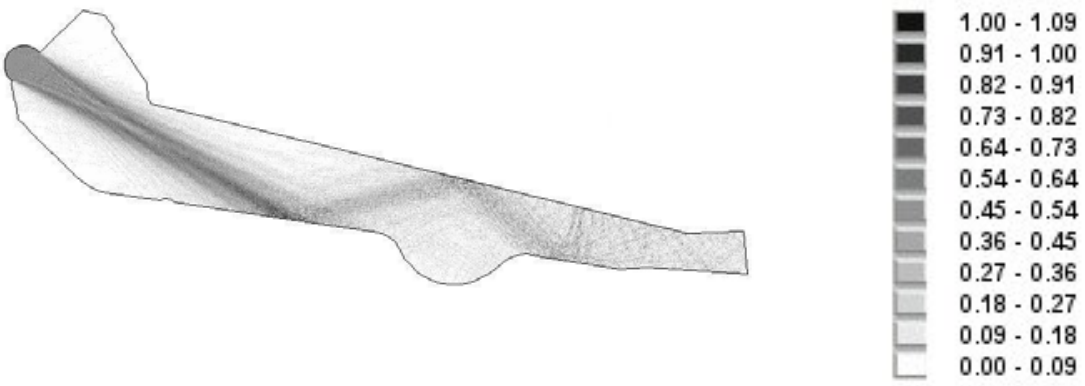

Figure 7. Hauteur de houle calculée sur un maillage de 7 millions de nouds.

Les résultats pour une onde courte se rapproche de l'optique géométrique avec un rayon qui se propage en zone éclairée et se réfléchit sur les ouvrages. Les résultats présentés sur les figures 6 et 7 avec les deux maillages (500 mille nœuds et 7 millions de nœuds) sont très proches. Le maximum de hauteur de houle atteint 1,03 - 1,02 - 1,03 - 1,07 - 1,09 m pour des maillages respectifs de 500 mille, 1 $2-4-7$ millions de nœuds, soit une erreur de $5 \%$ environ sur les maillages les moins denses.

Le nombre d'itérations (et donc le temps de résolution) pour le calcul avec une période de $3 \mathrm{~s}$ est sensiblement plus élevé que pour le calcul avec une période de $10 \mathrm{~s}$ (cf. paragraphe 4.3). 


\section{Conclusion}

Une nouvelle chaîne de calcul d'agitation de houle sur cluster a été mise en place. Allant du pré-traitement jusqu'au post-traitement en passant par un modèle d'équation de pente douce parallélisé, elle a été testée jusqu'à 28 millions de degrés de liberté répartis sur 10 machines. Les cas réels de grands bassins portuaires en France peuvent désormais être traités. Cette chaîne sur cluster permet d'augmenter la taille des domaines de calcul ou de réduire la période de houle étudiée. Le coût de résolution du problème dual augmentant avec le nombre de sous-domaines, un optimum en temps de calcul est trouvé pour 6 machines. Le temps de calcul est particulièrement réduit pour les calculs en houle aléatoire qui représentent quelques dizaines de calculs en houle régulière distribués sur les nœuds du cluster.

\section{Références bibliographiques}

1 BERKHOFF J.C.W. (1972). Computation of combined refraction and diffraction. Proceedings de la $13^{\text {ème }}$ Conférence internationale de Coastal Engineering, ASCE, pp 471-490

2 FARHAT C., LESOINNE M., LE TALLEC P., PIERSON K., RIXEN D. (2001). FETI-DP: a dual-primal unified FET method - part I: A faster alternative to the two-level FETI method. International Journal for numerical methods in Engineering. $\mathrm{n}^{\circ}$ 50, pp 1523-1544

${ }^{3}$ KAPYRIS G., KUMAR V. (1998). Metis a software package for partitioning unstructured graph, partitioning meshes, and computing fill-reducing orderings of sparses matrices. Technical report, University of Minnesota, Dept. of Comp. Sci.

${ }^{4}$ LEFRANCOIS E., BOUFFLET J-P., BIGOT S. (2004). Applications des techniques de calculs distribués à un code de calcul de houle aléatoire. $8^{\text {èmes }}$ Journées Nationales Génie Civil Génie Côtier. pp. 375-382

${ }^{5}$ LÖHNER R. (2001). Applied CFD techniques - An introduction based on Finite Element Methods. John Wiley, Chichester, 366 p.

${ }^{6}$ SERGENT P., ROPERT F., ORCEL O., HOUARI M., DUHAMEL D., DHATT G. (2002). Water waves in harbours areas: appreciation of open boundaries. Journal of Waterway, Port, Coastal and Ocean Engineering. n¹28 (5), pp 184189

7 TOSELlI A., WIDLUND O. (2005). Domain decomposition method Algorithm and theory. Springer-Verlag, Berlin, $450 \mathrm{p}$. 